\title{
Changes in Chemical Composition of Korean Red Ginseng (Panax ginseng C.A. Meyer) Extract With Alcohol Extraction
}

\author{
Kwang-Soon Shin ${ }^{1}$, Sung Hoon $\mathrm{Oh}^{2}$, Tae Young Kim ${ }^{3}$, Brian Yoon ${ }^{4}$, \\ Sung Sun Park, and Hyung Joo Suh $^{6 \dagger}$ \\ ${ }^{1}$ Department of Food Science and Biotechnology, Kyonggi University, Suwon 443-760, Korea \\ ${ }^{2}$ Department of Food and Biotechnology, Ansan College of Technology, Ansan 425-792, Korea \\ ${ }^{3}$ Bionic Trading Corporation, Ansan 425-906, Korea \\ ${ }^{4}$ Sejong Corporation, Incheon 405-849, Korea \\ ${ }^{5}$ Department of Food and Nutrition, Sungshin Women's University, Seoul 136-742, Korea \\ ${ }^{6}$ Department of Food and Nutrition, Korea University, Seoul 136-703, Korea
}

\begin{abstract}
We extracted red ginseng with various alcohol concentrations and evaluated total carbohydrate, uronic acid, polyphenols compounds and ginsenoside contents, and yields of alcohol extract. The water extraction $(0 \%$ alcohol extraction) showed a high level of total carbohydrate content. $10 \%$ and $20 \%$ alcohol extraction showed the highest uronic acid contents $(7,978.8$ and $7,872.7 \mu \mathrm{g} / \mathrm{mL}$ of extract, respectively). The efficiency order of the red ginseng extract (RGE) preparations in liberating polyphenols was: $0 \sim 50 \%$ alcohol $\geq 60 \%$ alcohol $>70 \sim 90 \%$ alcohol. Solid contents in RGE were decreased with increased alcohol concentration; the same tendency as with the results of total carbohydrate content. Total ginsenoside contents in $20 \sim 50 \%$ alcohol extracts showed similar levels $(42,962.9 \sim 47,930.8 \mu \mathrm{g} / \mathrm{mL}$ of extract). Water extraction showed the lowest ginsenoside content $(14,509.4 \mu \mathrm{g} / \mathrm{mL}$ of extract). The ginsenoside contents at above $60 \%$ alcohol were decreased with increased alcohol concentration. Generally, ginsenoside (Rg2, Rg1, Rf, Re, Rd, Rb2, Rc and Rb1) contents were increased with increased alcohol concentrations. However, Rg3 content was decreased with increases in alcohol concentration.
\end{abstract}

Key words: red ginseng, ginsenoside, polyphenols, uronic acid, extraction

\section{INTRODUCTION}

Ginsenosides are a class of steroid like compounds, particularly triterpenoid saponins, found exclusively in the roots of plant genus Panax (ginseng). Ginsenosides are attributed with cardio-protective, immunostimulatory, anti-fatigue, and hepatoprotective physiological and pharmacological effects (1-3). Commercially available ginseng is classified into fresh, white, and red ginseng. White ginseng is made by peeling the fresh ginseng roots and drying them without steaming. To preserve ginseng for an extended period of time, red ginseng is made by steaming and drying the fresh ginseng, suggesting chemical transformation by heat (4).

Recently, ginseng roots and their extracts have also become popular in the US and Europe as dietary health supplements and additives to foods and beverages. Extraction is the important step for the recovery of bioactive compounds from the plant raw materials. Extraction technologies must be versatile, relatively sim- ple and safe for the operating personnel and the consumers and inexpensive to use. Extraction methods are available for the cultivated and cultured cells of ginseng $(5,6)$. An increasingly common method of processing ginseng is to obtain an extract containing the ginsenosides by mixing dried ginseng powder with aqueous ethanolic solution, then removing the solid residue (7). These studies have recommended $40 \sim 50 \%$ ethanol for optimal extraction but they have only examined the effect on ginsenosides. However, changes of ginsenoside composition during alcoholic extractions from roots are not available currently.

Therefore, we have conducted extraction of various alcoholic concentrations and evaluated total carbohydrate, uronic acid, polyphenol contents and yields of alcohol extract. This paper describes the development of simple, convenient and optimal method of extraction using ethanol as solvent.

†Corresponding author. E-mail: suh1960@korea.ac.kr Phone: +82-2-940-2853, Fax: +82-303-344-2853 


\section{MATERIALS AND METHODS}

\section{Materials}

Six-year-old red ginseng was purchased at ginseng market in Geumsan, Korea. Standard ginsenosides including compound $\mathrm{K}$, Rh2, Rh1, Rg5, Rk1, Rg2, Rg3, $\mathrm{Rg} 1, \mathrm{Rf}, \mathrm{Re}, \mathrm{Rd}, \mathrm{Rb} 2, \mathrm{Rc}$ and $\mathrm{Rb} 1$ were purchased from Embo Lab. in Daejeon, Korea. All other chemicals were obtained from local suppliers and of reagent grade.

\section{Extraction of ginsenosides}

Extraction methods were based on the protocol of Ando et al. (8), but were simplified and modified for smaller samples. Red ginseng material $(5.0 \mathrm{~g})$ was placed in a soxhlet extractor and $100 \mathrm{~mL}$ of various alcoholic solutions were added into it. The ginseng was extracted twice, with the extract solution under reflux in a water bath at $70^{\circ} \mathrm{C}$, for $3 \mathrm{hr}$ each time. The combined extract was evaporated using a rotary evaporator under vacuum at $45^{\circ} \mathrm{C}$. The extract was used for the assay of total carbohydrate, uronic acid, polyphenols and dry weight. Ginsenosides were then assayed in the evaporated residue which was dissolved in $100 \mathrm{~mL}$ of distilled water and washed with $100 \mathrm{~mL}$ of diethyl ether. The aqueous layer was extracted 3 times with $100 \mathrm{~mL}$ of water-saturated $n$-butanol. The butanol solution was washed with $100 \mathrm{~mL}$ of distilled water to remove impurities, thereby obtaining crude saponins. The remaining butanolic solution was transferred to a tared round bottom flask for evaporation using a rotary evaporator under vacuum at $60^{\circ} \mathrm{C}$.

\section{HPLC analysis of ginsenosides}

The levels of 14 major ginsenosides were analyzed by an HPLC method developed by Kim et al. (9) and Li et al. (10). The HPLC system used an ACME 9000 HPLC (Young Lin Instrument Co., LTD, Anyang, Korea) with an ELSD detector. A prevail carbohydrate ES column $(4.6 \times 250 \mathrm{~mm}$, Alltech Associates, Inc., Illinois, USA) was also used. The solvent flow rate was held constant at $0.8 \mathrm{~mL} / \mathrm{min}$. The column temperature was fixed at $35^{\circ} \mathrm{C}$ using a column oven. The mobile phase used for the separation consisted of solvent $\mathrm{A}$ (acetonitrile : water : IPA=80:5:15) and solvent $\mathrm{B}$ (acetonitrile : water : IPA=67:21:12). A gradient elution procedure was used as $0 \sim 28 \mathrm{~min} 90 \% \mathrm{~A}, 28 \sim 35 \mathrm{~min} 15 \%$ A, $35 \sim 45 \min 20 \%$ A, $45 \sim 50 \min 25 \%$ A, $50 \sim 51$ $\min 10 \% \mathrm{~A}, 51 \sim 57 \min 0 \% \mathrm{~A}, 57 \sim 58 \min 75 \% \mathrm{~A}$, and $58 \sim 65 \mathrm{~min} 90 \% \mathrm{~A}$. The injection volume was 20 $\mu \mathrm{L}$ for analysis. Peak identifications were based on retention times and comparisons with injected standard samples. All solutions were filtered through $0.45 \mu \mathrm{m}$ membrane syringe filters (Millipore Co.) before analysis.
To determine calibration curves, the ginsenoside standards compound $\mathrm{K}, \mathrm{Rh} 2$, Rh1, Rg5, Rk1, Rg2, Rg3, Rg1, $\mathrm{Rf}, \mathrm{Re}, \mathrm{Rd}, \mathrm{Rb} 2, \mathrm{Rc}$ and $\mathrm{Rb} 1$ were dissolved individually in HPLC-grade methanol, whereupon calibration standards were prepared by mixing different concentrations in appropriate quantities. The level of total ginsenosides was determined by summing the levels of the 14 ginsenosides.

\section{Analytical methods}

Total polyphenol (TP) content was determined using the Folin-Ciocalteu method (11), adapted to a microscale using gallic acid as the standard $(50 \sim 800 \mu \mathrm{g} / \mathrm{mL})$. Total carbohydrate and uronic acid were determined by the phenol- $\mathrm{H}_{2} \mathrm{SO}_{4}$ (12) and $m$-hydroxydiphenyl (13) method, respectively, using glucose and galacturonic acid as the respective standards. In all cases, analyses were performed in triplicate, unless elsewhere specified, and values averaged. The standard deviation (SD) was also calculated. All data were analyzed by one-way analysis of variance and Duncan's multiple range tests using the SPSS version 10.0 software (SPSS, Chicago, IL). Results were considered significant at $\mathrm{p}<0.05$.

\section{Bittemess evaluation}

The extract obtained by the above extraction methods were made up to exactly $20 \mathrm{~mL}$ with purified water. The suspension was used to evaluate the bitterness. Sensory evaluations of bitterness were conducted in triplicate by the panels, using a five-point hedonic scale method (14). The bitter taste was evaluated as follows: very low (1 point), low ( 2 point), medium ( 3 point), strong (4 point), and very strong (5 point). The results of the sensory evaluations were expressed as the means \pm standard deviation (SD) of 10 panelists. The significance was verified via Duncan's multiple range tests, using the SPSS software package.

\section{RESULTS AND DISCUSSION}

\section{Total carbohydrate content of the red ginseng extract (RGE)}

Total carbohydrate content of the RGE is shown in Fig. 1. The water extraction ( $0 \%$ alcohol extraction) showed a high level of total carbohydrate content. Total carbohydrate decreased as alcoholic concentrations increased. However, there was not a significant difference in total carbohydrate between $10 \%$ and $60 \%$ alcohol $(\mathrm{p}<0.05)$. $70 \% \sim 90 \%$ of alcohol extractions also had similar total carbohydrate concentrations.

The major components of Korean ginseng are carbohydrates, which include starch, polysaccharides, cellulose, and glycosides. Ginseng mainly consists of carbo- 


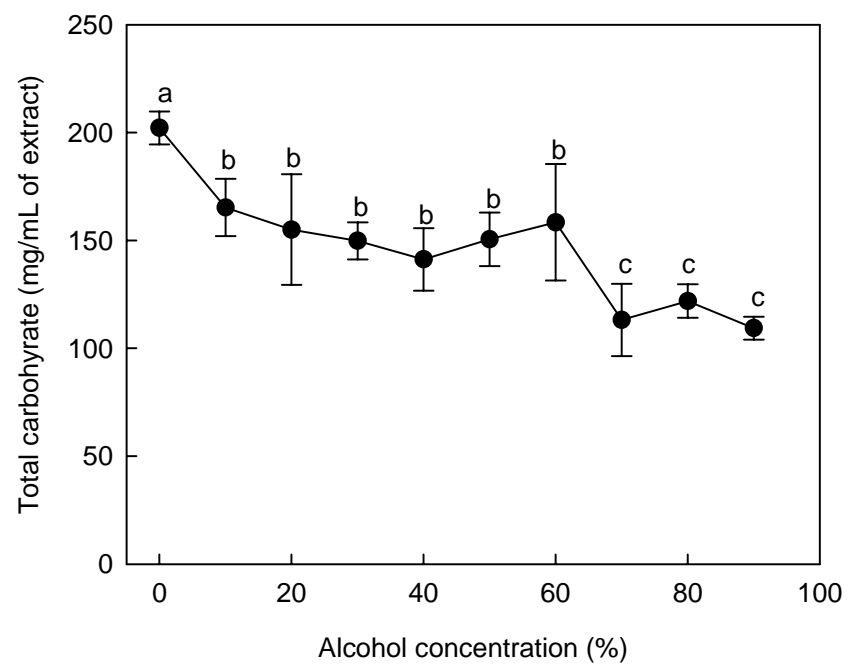

Fig. 1. Changes in total carbohydrate contents of Korean red ginseng extracted with various alcohol concentrations. Different letters at each measurement indicate significant differences among groups $(\mathrm{p}<0.05)$.

hydrates $(60 \sim 70 \mathrm{~g}$ carbohydrate/100 g solid) and starch is a major component of ginseng carbohydrates (15). Red ginseng with inner white showed not only less dense tissue structure but also relatively small amounts of starch (16). The occurrence of inner hole and inner white in red ginseng increased when the amount of starch in fresh ginseng decreased (17). Therefore, water extraction showed a large amount of total carbohydrate contents because of water solubility.

\section{Uronic acid content of RGE}

Uronic acid (as acidic polysaccharide) contents of RGEs are shown in Fig. 2. 10\% and 20\% alcohol extraction showed the highest uronic acid content $(7,978.8$

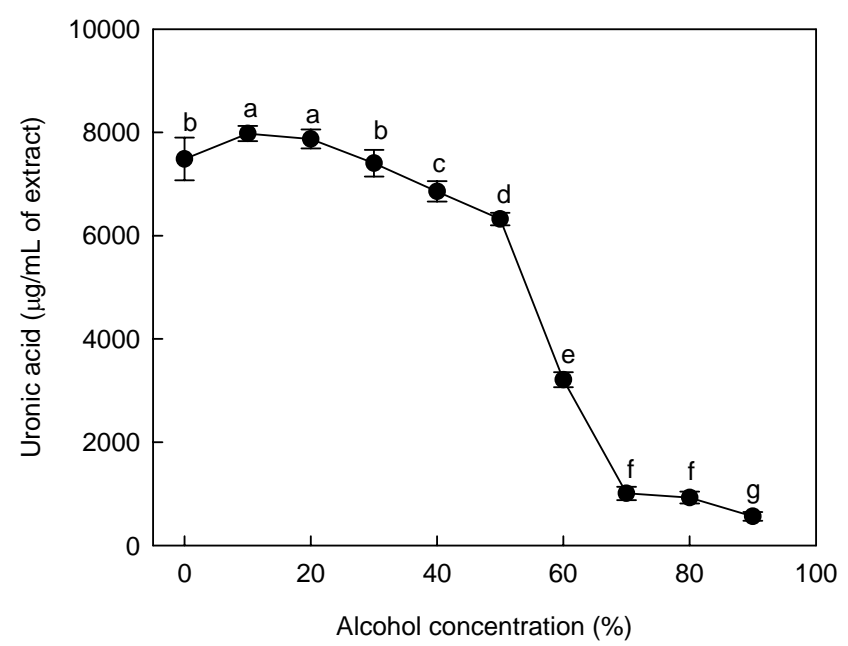

Fig. 2. Changes in uronic acids contents of Korean red ginseng during extracted with various alcohol concentrations. Different letters at each measurement indicate significant differences among groups $(\mathrm{p}<0.05)$. and 7,872.7 $\mu \mathrm{g} / \mathrm{mL}$ of exract, respectively). The uronic acid contents were decreased with alcohol concentrations above $30 \%$. Especially, the contents were sharply decreased at alcohol concentration above 50\%.

Panax ginseng may have a pectin-type polysaccharide mainly composed of galacturonic and glucuronic acids (93\%). This uronic acid content appears to be significantly higher than previously reported (18), and this can probably be attributed to improved techniques for the separation and calculation of neutral and uronic acids. Uronic acids obtained from plant sources have been shown to exhibit a variety of biological activities, including immunostimulatory, antioxidant, antitumor, and antiviral properties (19). A uronic acid with immunomodulating activity, which was obtained from $P$. ginseng leaves, was found to consist of a highly branched glycan structure, composed of arabinose, galactose, rhamnose and galacturonic acid with a $\beta-(1,3)$ linked galactan backbone (20).

\section{Polyphenol and solid contents of RGE}

Polyphenols are considered secondary metabolites that are synthesized in plants and function as a defense mechanism in response to various stress conditions (21). The observed increase in total polyphenols in roots of $P$. ginseng was accompanied by increased flavonoid content, total proteins and antioxidant activity. Induced polyphenols accumulation may also have an impact on biological activities of ginseng.

As shown in Fig. 3, the efficiency order of the RGE preparations in liberating polyphenols were: $0 \sim 50 \%$ alcohol $\geq 60 \%$ alcohol $>70 \sim 90 \%$ alcohol. There were not a significantly difference of polyphenol contents between

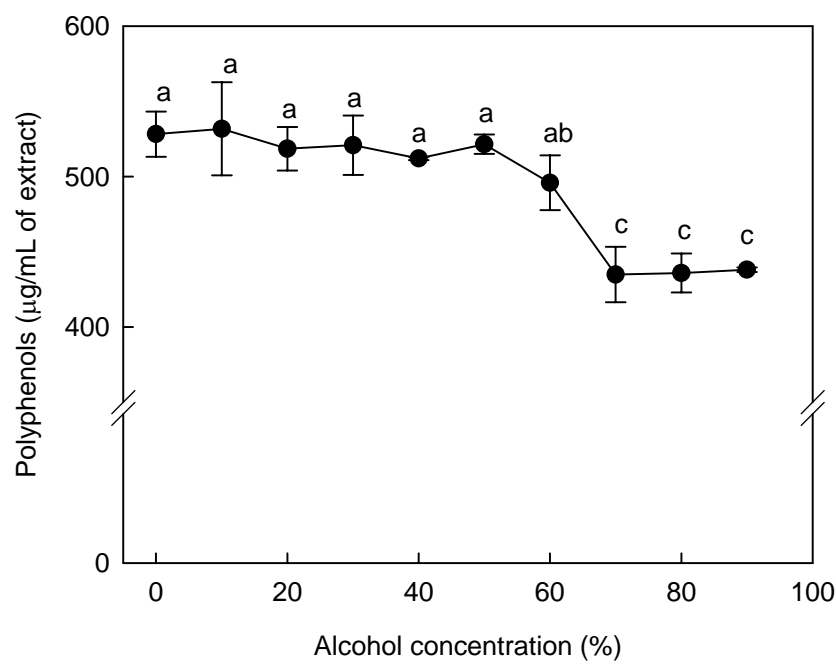

Fig. 3. Changes in polyphenols contents after extraction of Korean red ginseng with various alcohol concentrations. Different letters at each measurement indicate significant differences among groups $(p<0.05)$. 
$0 \sim 50 \%$ and $60 \%$ alcohol extraction. Polyphenols contents tended to decrease with increased alcohol concentration. In general, polyphenols were extracted very well with about $70 \%$ alcohol, but alcohol extraction below $50 \%$ showed a higher level of polyphenols than alcohol extraction at above $50 \%$. The polyphenols in ginseng were assumed to have high contents of glycoside type polyphenols on the basis of high extraction level by alcohol extraction below $50 \%$. The major components of ginseng are ginsenosides, which are glycosides with a dammarane skeleton aglycone (22).

Besides saponin, $P$. ginseng also accumulates other secondary metabolites (phenolic compounds), and the growth of $4 \sim 6$ years is needed for proper accumulation of secondary metabolites. Tissue culture is an important tool of plant biotechnology and one of its potential applications is for the production of valuable plant secondary metabolites $(21,23)$. And therefore, development of an efficient root culture system for commercial production of ginseng root requires integrated enhancement strategies and increased extraction yields of polyphenols.

As shown in Fig. 4, solid contents also showed the same tendency with total carbohydrate contents. The contents showed a decreasing tendency with increases in alcohol concentration. However, there were not a significant difference of solid contents between $20 \%$ and $60 \%$ alcohol. Above results indicate that water extraction increases the solid content due to increases of total carbohydrate, acid polysaccharide and polyphenols contents.

\section{Ginsenoside composition of RGE}

Ginseng saponins (ginsenosides) are the principal components having pharmacological and biological activities, such as antidiabetic, and anti-tumor activities (24). More than 30 different ginsenosides so far have been isolated and identified from ginseng saponins.

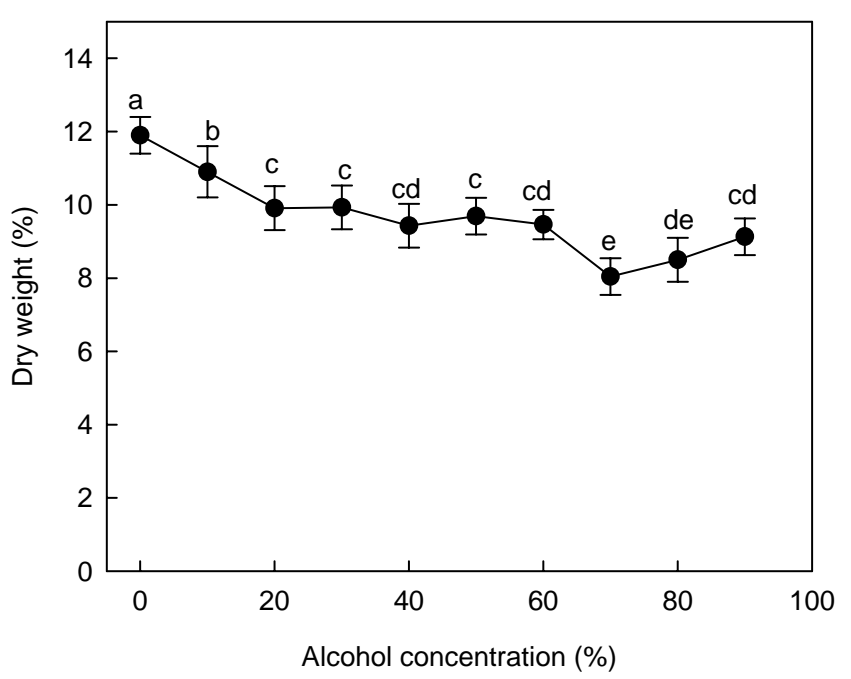

Fig. 4. Changes in solid contents after extraction of Korean red ginseng with various alcoholi concentrations. Different letters at each measurement indicate significant differences among groups $(\mathrm{p}<0.05)$.

Ginsenoside compositions of RGEs are shown in Table 1. Total ginsenoside contents in $20 \sim 50 \%$ alcohol extracts showed similar levels $(42,962.9 \sim 47,930.8 \mu \mathrm{g} /$ $\mathrm{mL}$ of extract). Water extraction showed the lowest ginsenoside content $(14,509.4 \mu \mathrm{g} / \mathrm{mL}$ of extract). The contents at above $60 \%$ alcohol were decreased with increase of alcohol concentration. Almost all ginsenoside ( $\mathrm{Rg} 2$, $\mathrm{Rg} 1$, Rf, Re, Rd, Rb2, Rc and Rb1) contents were increased with increased alcohol concentration. However, Rg3 content was decreased with increases of alcohol concentration.

Extraction trials were carried out at room temperature or using heat (25) or sonication (26) to enhance recovery of ginsenosides. The use of heat in the extraction procedure was proven to degrade the thermally unstable malonyl-ginsenosides into the corresponding neutral ginse-

Table 1. Changes of ginsenoside compositions during extraction with various alcohol concentration from Korean red ginseng

\begin{tabular}{|c|c|c|c|c|c|c|c|c|c|c|}
\hline \multirow[b]{2}{*}{ Ginsenoside } & \multicolumn{10}{|c|}{ Concentration $(\mu \mathrm{g} / \mathrm{mL}$ of extract) } \\
\hline & Water & $\begin{array}{c}10 \% \\
\text { EtOH }\end{array}$ & $\begin{array}{c}20 \% \\
\text { EtOH } \\
\end{array}$ & $\begin{array}{l}30 \% \\
\text { EtOH }\end{array}$ & $\begin{array}{c}40 \% \\
\text { EtOH } \\
\end{array}$ & $\begin{array}{c}50 \% \\
\text { EtOH } \\
\end{array}$ & $\begin{array}{c}60 \% \\
\text { EtOH } \\
\end{array}$ & $\begin{array}{c}70 \% \\
\text { EtOH }\end{array}$ & $\begin{array}{c}80 \% \\
\text { EtOH }\end{array}$ & $\begin{array}{c}90 \% \\
\text { EtOH } \\
\end{array}$ \\
\hline Compd K & 0 & 0 & 0 & 0 & 0 & 0 & 0 & 0 & 0 & 0 \\
\hline $\mathrm{Rh} 2$ & 0 & 0 & 0 & 0 & 0 & 0 & 0 & 0 & 0 & 0 \\
\hline Rh1 & 118.8 & 115.1 & 113.4 & 116.4 & 118.4 & 112.1 & 122.3 & 0 & 0 & 0 \\
\hline Rg5+RK1 & 1850.7 & 2293.9 & 1336.8 & 407.5 & 410.4 & 1607.8 & 1245.8 & 389.3 & 585.6 & 701.5 \\
\hline Rg2 & 340.2 & 538.1 & 582.5 & 538.1 & 521.8 & 521.6 & 429.1 & 304.2 & 280.0 & 359.2 \\
\hline Rg3 & 453.6 & 495.8 & 317.4 & 219.5 & 196.6 & 314.6 & 245.6 & 176.4 & 163.4 & 206.4 \\
\hline Rg1 & 348.5 & 1063.3 & 1359.6 & 1241.4 & 1232.3 & 1120.2 & 861.8 & 509.6 & 419.1 & 684.7 \\
\hline $\mathrm{Rf}$ & 282.5 & 697.6 & 895.6 & 790.3 & 766.2 & 770.7 & 563.4 & 402.1 & 291.6 & 469.6 \\
\hline $\operatorname{Re}$ & 1576.0 & 4961.1 & 6717.5 & 6085.8 & 6155.4 & 5464.7 & 4241.4 & 2704.5 & 2076.7 & 3422.5 \\
\hline $\mathrm{Rd}$ & 1457.7 & 4434.5 & 5894.5 & 5617 & 5945.9 & 5636.2 & 3810.3 & 2492.4 & 1882.4 & 3059.2 \\
\hline $\mathrm{Rb} 2$ & 1192.3 & 3000.5 & 3829.3 & 3459 & 3474.9 & 3613.6 & 2821.9 & 2066.4 & 1445.0 & 2358.5 \\
\hline $\mathrm{Rc}$ & 3122.8 & 8502.6 & 10967.6 & 10195.9 & 10168.3 & 10323.5 & 7802.5 & 5048.2 & 3746.2 & 6544.0 \\
\hline Rb1 & 3766.3 & 12577.8 & 15916.6 & 14292.0 & 14226.3 & 14382.0 & 11164.1 & 6839.3 & 4660.5 & 8742.2 \\
\hline Total & 14509.4 & 38680.3 & 47930.8 & 42962.9 & 43216.5 & 43867.0 & 33308.2 & 20932.4 & 15550.5 & 26547.8 \\
\hline
\end{tabular}


nosides. Court et al. (27) showed that, while partial degradation $(50 \%)$ occurred after $5 \mathrm{hr}$ of extraction using methanol in a soxhlet apparatus, a minimum of $20 \mathrm{hr}$ were necessary to achieve total conversion. Preparation of Korean red ginseng relies on steam processing to ensure preservation which may alter the ginsenoside composition. Steaming ginseng has been shown to produce ginsenosides that are not present in raw ginseng (25). Differently from white ginseng, which is obtained from the dried roots of $P$. ginseng, red ginseng is produced by steaming and drying up the roots of $P$. ginseng. This heating procedure causes degradation of the malonyl ginsenosides $\mathrm{m}-\mathrm{Rb} 1, \mathrm{~m}-\mathrm{Rb} 2$ and $\mathrm{m}-\mathrm{Rc}$ and $\mathrm{m}-\mathrm{Rd}$ in the ginsenosides $\mathrm{Rb} 1, \mathrm{Rb} 2, \mathrm{Rc}$ and $\mathrm{Rd}$, respectively. Ginseng root and leaf hot water reflux extract were found to have 23 and 19 different ion fragments, respectively, whereas, the $80 \%$ aqueous ethanol extract contained 14 fragments. Furthermore, the $80 \%$ aqueous ethanol extract contained a malonyl ginsenoside that was not present in the hot water reflux extract. Malonyl ginsenosides are heat-labile and readily demalonylate (28).

However, studies on the effect of drying of American ginseng, based on changes in the neutral ginsenosides, resulted in recommended drying temperatures in the range $20 \sim 50^{\circ} \mathrm{C}(29)$.

Zhang et al. (30) reported the effect of ethanol concentration on the extraction yield of ginsenoside from $P$. quinquefolium L. root (American ginseng). The extraction yield of ginsenoside was improved by increasing ethanol concentration in the range of $10 \sim 70 \%$. When the ethanol concentration is higher than $70 \%$, the extraction yield of ginsenoside decreased slowly with increasing of ethanol concentration. It is known that the solubility of neutral and malonyl ginsenosides are varied in different concentrations of ethanol. Therefore, the breakage degree of the cell membrane is different in different concentration ethanol. The protein could be coagulated in higher concentrations of ethanol, making larger diffusion resistance. It was reported (31) that the maximum extraction of neutral, malonyl, and total ginsenosides was obtained with $70 \%, 40 \%$, and $60 \%$ ethanol, respectively.

However, our results showed $20 \sim 50 \%$ ethanol as optimal extraction concentrations. This discrepancy could be due to the quality variation of products by manufacturer, different drying methods of red ginseng and American ginseng, and genotype-dependent variability in different species of ginseng.

\section{Bittemess evaluation of RGE}

Sensory scores of the RGE extracted with various alcohol concentrations are shown in Fig. 5. The bitterness

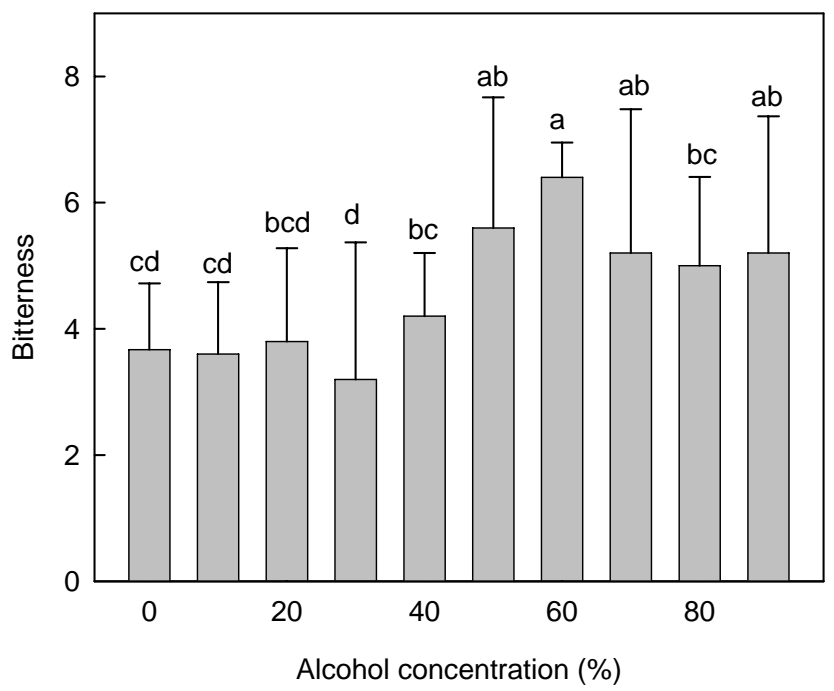

Fig. 5. Changes in bitterness of Korean red ginseng following extraction with various alcoholic concentration. Different letters at each measurement indicate significant differences among groups $(\mathrm{p}<0.05)$.

values of RGE extracted between 0 and $30 \%$ alcohol showed 3.2 3.8, and those of RGE extracted with alcohols above 50\% showed 5.0 6.4. Although ginsenoside contents of RGE extracted between 0 and $30 \%$ alcohol showed high levels, the bitterness of the RGE extract might be decreased due to increase of total carbohydrate contents.

Saponins are non-volatile, amphiphilic compounds that occur in a wide variety of legume seeds such as peas, soybeans, lentils and lupins (32). They are chemically referred to as triterpene glycosides, and consist of non-polar aglycones coupled with one or more sugar chains (33). A large number of different saponins may occur within a single plant species (34). Ginsenosides are triterpenes saponins considered to be the main bioactive principles of the most important Oriental herbal medicine "ginseng" derived from the roots and rhizomes of different Panax species. The bitterness of peas, as well as that of soybeans, has been ascribed to the presence of saponins (35).

Sweetness suppression of bitterness has been demonstrated for sucrose and quinine mixtures and for sucrose and caffeine mixtures (36). An increase in solution viscosity decreased the bitterness of quinine and other solutions (37). Because isoflavones and saponins bind to soy proteins through hydrophobic interactions (38), it seems likely that these phytochemicals bind to milk proteins, and a reduction in bitterness may result. Robinson and others (39) reported using starch to suspend isoflavone aglycons in water with resulting thresholds in the $\mathrm{mM}$ range, but not much higher than our thresholds for isoflavones in milk, also supporting the hydrophobic 
binding of the saponins to the hydrophobic regions of native starch.

Therefore, RGE extracted between with alcohol concentrations between 0 and $30 \%$ alcohol might have high contents of starch, and the bitterness might be reduced owing to the binding of the saponins to the hydrophobic regions of native starch.

\section{ACKNOWLEDGEMENT}

We thank the Sejong Corporation, Korea for financial support of this work.

\section{REFERENCES}

1. Attele AS, Wu JA, Yuan CS. 1999. Ginseng pharmacology: multiple constituents and multiple action. Biochem Pharmacol 58: 1685-1693.

2. Gillis CN. 1997. Panax ginseng pharmacology: a nitric oxide link. Biochem Pharmacol 54: 1-8.

3. Shin HR, Kim JY, Yun TK, Morgan G, Vainio H. 2000. The cancer-preventive potential of Panax ginseng: a review of human and experimental evidence. Cancer Cause Control 11: 565-576.

4. Park JD. 1996. Recent studies on the chemical constituents of Korean ginseng (Panax ginseng C.A. Meyer). Kor J Ginseng Sci 20: 389-415.

5. Kwon JH, Lee GD, Belanger JMR, Pare JRJ. 2003. Effect of ethanol concentration on the efficiently of extraction of ginseng saponins when using a microwave-assisted process (MAPTM). Int J Food Sci Technol 38: 615-622.

6. Kwon JH, Kim KE, Lee GD. 2000. Optimization of microwave-assisted extraction under atmospheric pressure condition for soluble ginseng components. Korean J Food Sci Technol 32: 117-124.

7. Xu W, Zheng Q, Zhou H, Zhao Q. 1996. Studies of extraction technology on saponins in Asian ginseng residue. Xiandai Yingyong Yaoxue 13: 34-35.

8. Ando T, Tanaka O, Shibata S. 1971. Comparative studies on the saponins and sapogenins ginseng and related crude drugs. Syoyakugaku Zasshi 25: 28-32.

9. Kim SN, Ha YW, Shin H, Son SH, Wu SJ, Kim YS. 2007. Simultaneous quantification of 14 ginsenosides in Panax ginseng C.A. Meyer (Korean red ginseng) by HPLC-ELSD and its application to quality control. $J$ Pharm Biomed Anal 45: 164-170.

10. Li J, Qi H, Qi LW, Yi L, Li P. 2007. Simultaneous determination of main phytoecdysones and triterpenoids in radix achyranthis bidentatae by high-performance liquid chromatography with diode array-evaporative light scattering detectors and mass spectrometry. Anal Chim Acta 596: 264-272.

11. Waterman PG, Mole S. 1994. Analysis of phenolic plant metabolites. Blackwell Scientific Publications, Oxford, UK. p 83-91.

12. Dubois M, Gilles KA, Hamilton JK, Rebers PA, Smith F. 1956. Colorimetric method for determination of sugars and related substances. Anal Chem 28: 350-356.

13. Blumenkrantz N, Asboe-Hansen G. 1973. New method for quantitative determination of uronic acids. Anal Biochem 54: 484-489.
14. Herbert A, Joel LS. 1993. Sensory evaluation practices. Academic Press, New York, USA. p 68-75.

15. Ko SR, Choi KJ, Han KW. 1996. Comparison of proximate composition, mineral nutrient, amino acid and free sugar contents of several Panax species. Korean J Ginseng Sci 20: 36-41.

16. Do JH, Kim SD, Sung HS. 1985. Biochemical and histological characteristics of inferior red ginseng. Korean $J$ Ginseng Sci 9: 256-263.

17. Oh HI, Lee SJ, Do JH, Kim SD, Hong SK. 1981. Physical and chemical characteristics of Panax ginseng starch. Korean J Ginseng Sci 5: 114-121.

18. Lee JH, Park EK, Uhm CS, Chung MS, Kim KH. 2004. Inhibition of Helicobacter pylori adhesion to human gastric adenocarcinoma epithelial cells by acidic polysaccharides from Artemisia capillaris and Panax ginseng. Planta Med 70: 615-619.

19. Nangia-Marker P, Conklin J, Hogan V, Raz A. 2002. Carbohydrate-binding proteins in cancer, and their ligands as therapeutic agents. Trends Mol Med 8: 187-192.

20. Tomoda M, Hirabayashi K, Shimizu N, Gonda R, Ohara N. 1994. The core structure of ginsenan PA, a phagocytosis-activating polysaccharide from the root of Panax ginseng. Biol Pharm Bull 17: 1287-1291.

21. Dixon RA, Paiva NL. 1995. Stress-induced phenylpropanoid metabolism. Plant Cell 7: 1085-1097.

22. Tanaka N, Tanaka O, Shibata S. 1972. Chemical studies on the oriental plant drugs. XXVIII. Saponins and sapogenins of ginseng; Stereochemistry of sapogenin of ginsenoside Rb1, Rb2 and Rc. Chem Pharm Bull 20: 1212-1216.

23. Raag H, Kuhn DN, Kahlbroeck K. 1984. Coordinated regulation of coumarate: $\mathrm{CoA}$ ligase and phenylalanine ammonia lyase mRNA in cultured plant cells. $\mathrm{J}$ Biol Chem 256: $52-60$.

24. Park JD, Rhee DK, Lee YH. 2005. Biological activities and chemistry of saponins from Panax ginseng C.A. Meyer. Phytochem Rev 4: 159-175.

25. Kim WK, Kim JM, Han SB, Lee SK, Kim ND, Park MK, Kim CK, Park JH. 2000. Steaming of ginseng at high temperature enhances biological activity. J Nat Prod 63: 1702-1704.

26. Fuzzati N, Gabetta B, Jayakar K, Pace R, Ramaschi G, Villa F. 2000. Determination of ginsenosides in Panax ginseng roots by liquid chromatography with evaporative light-scattering detection. J AOAC Int 83: 820-829.

27. Court WA, Hendel JG, Elmi J. 1996. Reversed-phase high performance liquide chromatography determination of ginsenosides of Panax quinquefolium. J Chromatogr A 755: 11-17.

28. Yamaguchi H, Kasai R, Matsuura H, Tanaka O, Fuwa T. 1988. High-performance liquid chromatographic analysis of acidic saponins of ginseng and related plants. Chem Pharm Bull 36: 3468-3473.

29. Wang T, Jia Z, Liu C, Ren G. 1990. The studies of processing technology and technique on American ginseng. Zhongguo Yaoxue Zazhi 15: 24-27.

30. Zhang S, Chen R, Wu H, Wang C. 2006. Ginsenoside extraction from Panax quinquefolium L. (American ginseng) root by using ultrahigh pressure. J Pharm Biomed Anal 41: 57-63.

31. Du XW, Wills RBH, Stuart DL. 2004. Changes in the neutral and malonyl ginsenosides of American ginseng roots (Panax quinquefolium) were examined during dry- 
ing, storage and extraction. Food Chem 86: 155-159.

32. Lasztity R, Hidvegi M, Bata A. 1988. Saponins in food. Food Rev Int 14: 371-390.

33. Oleszek WA. 2002. Chromatographic determination of plant saponins. J Chromatogr A 967: 147-162.

34. Price KR, Eagles J, Fenwick GR. 1988. Saponin composition of 13 varieties of legume seed using fast atom bombardment mass spectrometry. J Sci Food Agric 42: 183193.

35. Price KR, Griffiths NM, Curl CL, Fenwick GR. 1985. Undesirable sensory properties of the dried pea (Pisum sativum): the role of saponins. Food Chem 17: 105-115.

36. Calvino AM, García-Medina MR, Cometto-Munoz JE. 1990. Interactions in caffeine sucrose and coffee sucrose mixtures: evidence of taste and flavor suppression. Chem Senses 15: 505-519.

37. Burns DJW, Noble AC. 1985. Evaluation of the separate contribution of viscosity and sweetness of sucrose to perceived viscosity, sweetness and bitterness of vermouth. $J$ Texture Stud 16: 365-381.

38. Rickert DA, Johnson LA, Murphy PA. 2004. Improved fractionation of glycinin and beta-conglycinin and partitioning of phytochemicals. J Agric Food Chem 52: 17261734.

39. Robinson KM, Klein BP, Lee SY. 2004. Utilizing the $\mathrm{R}$-index measure for threshold testing of model soy isoflavone solutions. J Food Sci 69: SNQ1-4.

(Received July 16, 2008; Accepted August 2, 2008) 